\title{
Analysis of association gates in radar tracking based on Kalman filter
}

Krzysztof Jaroś, Wojciech Buda

Krzysztof Jaroś, Wojciech Buda, "Analysis of association gates in radar tracking based on Kalman filter," Proc. SPIE 11442, Radioelectronic Systems Conference 2019, 1144206 (11 February 2020); doi: 10.1117/12.2565276

SPIE. Event: Radioelectronic Systems Conference 2019, 2019, Jachranka, Poland 


\title{
ANALYSIS OF ASSOCIATION GATES IN RADAR TRACKING BASED ON KALMAN FILTER
}

\author{
Krzysztof Jarośa and Wojciech Buda ${ }^{\mathrm{a}}$ \\ aPIT-RADWAR S.A., Hallera 233A, Gdańsk, Poland
}

\begin{abstract}
Radar target tracking can be presented as combination of track filtering and association algorithm, where association problem is to determine validity of observation-to-track pairing. Track represents single real object build as set of observations connected together using association process. To eliminate unlikely observation-to-track pairings association algorithms employ gating techniques. Using track filtering results, a gate is formed, as a constricted space around tracked object predicted position. This space is called tracking gate and all observation that fall within the gate are considered for the track update. In this paper analysis of tracking gate shape and behaviour is investigated, with association gates build using Kalman filtering and Mahalanobis distance as a metric. Results are presented as graphical spatial imaging of tracking gates, for simulated tracking data considering different manoeuvring scenarios and tracking filter parametrisation.
\end{abstract}

Keywords: Association gate, Mahalanobis distance, Kalman filter

\section{INTRODUCTION}

Growing requirements for modern radar systems, regarding tracking of closely spaced manuvering targets in cluttered environment, creates new challenges in the field of radar target tracking applications. One of the main issues is validation of observation-to-track pairing. The goal is to distinguish correct and false detections in the process of automatic track initiation and maintenance. Existing association algorithms (e.g. PDA, JPDA, MHT) that aim at creating a robust system, resistant to clutter, jamming and measurement errors, faces a issue of significant computational cost ${ }^{1,2,3}$. To solve the problem of insufficient computing power, tracking systems use a number of techniques of increasing complexity, whose task is to limit the number of performed association operations. In practical solutions, the final stage of that process is gating algorithm. Gating can be described as an assessment of a degree of correlation between the measurement and predicted state of the tracked object. Observation that satisfy gating relationship is considered for track update. Gating algorithms use results of tracking filter to build a decision mechanism. This paper concerns ellipsoidal gates defined by the statistical distance between the measurement and the predicted state of the object, compared with the acceptance threshold. The goal of the study is to analyse and visualize the shapes and evolution of association gates in space depending on the selected group of factors and parameters of the tracking process. The analysis covers the differences in gate sizes resulting from the parametrization of the tracking filter, the evolution of the shape of the association gates depending on the state vector of the tracked object and the change of the size of the association gates in the event of extrapolation of the track in the absence of a detection.

\section{TRACKING FILTER}

\subsection{Kalman filter}

Practical solutions of modern radar tracking systems are usually based on the Kalman filter. The Kalman filter is an optimal estimator in the sense of minimizing mean square prediction error for linear objects. ${ }^{4}$ The Kalman algorithm assumes that the object model and measurement model are described by linear equations, and the random processes used during modeling are Gaussian, and are described by normal distributions with known

Further author information: (Send correspondence to Krzysztof Jaros, Wojciech Buda)

A.A.A.: E-mail: Krzysztof.Jaros@pitradwar.com

E-mail: Wojciech.Buda@pitradwar.com

Radioelectronic Systems Conference 2019, edited by Piotr Kaniewski, Jan Matuszewski, Proc. of SPIE Vol. 11442, $1144206 \cdot$ (c) 2020 SPIE · CCC code: 0277-786X/20/\$21 · doi: 10.1117/12.2565276 
mean values and covariances. The discrete-time Kalman algorithm to describe dynamic and measurement model use following equations:

$$
\begin{gathered}
x(k+1)=F x(k)+G n(k), \\
y(k)=H x(k)+v(k),
\end{gathered}
$$

Where:

- $k$ - the time index

- $x(k)$ - the states vector

- $F$ - the station transition model

- $G$ - is the noise model which is applied to the noise vector $n(k)$

- $H$ - the observation model

- $n(k)$-is the process noise which is assumed to be drawn from a zero mean multivariate normal distribution with covariance $Q$

- $y(k)$ - measurements vector

- $v(k)$ - is the observation noise which is assumed be drawn from a zero mean multivariate normal distribution with covariance $R$

1 Update:

$$
\begin{gathered}
\tilde{y}(k)=y(k)-H \widehat{x}(k \mid k-1), \\
S(k)=H P(k \mid k-1) H^{T}+R, \\
K(k)=P(k \mid k-1) H^{T} S^{-1}(k), \\
\widehat{x}(k \mid k)=\widehat{x}(k \mid k-1)+K(k) \tilde{y}(k), \\
P(k \mid k)=P(k \mid k-1)-K(k) S(k) K^{T}(k),
\end{gathered}
$$

2 Prediction

$$
\begin{gathered}
\widehat{x}(k+1 \mid k)=F x(k \mid k), \\
P(k+1 \mid k)=F P(k \mid k) F^{T}+G Q G^{T},
\end{gathered}
$$

Where:

- $\tilde{y}(k)$ - the innovation vector

- $S(k)$ - the covariance of the innovation

- $K(k)$ - the optimal Kalman gain matrix

- $x(k \mid k)$ - updated vector of state

- $P(k \mid k)$ - updated estimate covariance

- $R$ - the covariance of the observation noise

- $\widehat{x}(k+1 \mid k)$ - vector of predicted state

- $P(k+1 \mid k)$ - predicted error covariance 
- $Q$ - the covariance of the process noise

For this paper the state vector is written as $x(k)=\left[x, y, z, v_{x}, v_{y}, v_{z}, a_{x}, a_{y}, a_{z}\right]^{T}$ where $x, y, z$ describe position, $v_{x}, v_{y}, v_{z}$ velocity and $a_{x}, a_{y}, a_{z}$ acceleration in carthesian coordinate system respectively. Following matrices were used to describe the state space model:

$$
\begin{aligned}
& F=\left|\begin{array}{ccccccccc}
1 & 0 & 0 & T & 0 & 0 & T^{2} / 2 & 0 & 0 \\
0 & 1 & 0 & 0 & T & 0 & 0 & T^{2} / 2 & 0 \\
0 & 0 & 1 & 0 & 0 & T & 0 & 0 & T^{2} / 2 \\
0 & 0 & 0 & 1 & 0 & 0 & T & 0 & 0 \\
0 & 0 & 0 & 0 & 1 & 0 & 0 & T & 0 \\
0 & 0 & 0 & 0 & 0 & 1 & 0 & 0 & T \\
0 & 0 & 0 & 0 & 0 & 0 & 1 & 0 & 0 \\
0 & 0 & 0 & 0 & 0 & 0 & 0 & 1 & 0 \\
0 & 0 & 0 & 0 & 0 & 0 & 0 & 0 & 1
\end{array}\right|, \\
& H=\left|\begin{array}{lllllllll}
1 & 0 & 0 & 0 & 0 & 0 & 0 & 0 & 0 \\
0 & 1 & 0 & 0 & 0 & 0 & 0 & 0 & 0 \\
0 & 0 & 1 & 0 & 0 & 0 & 0 & 0 & 0
\end{array}\right|, \\
& G=\left|\begin{array}{ccc}
T^{2} / 2 & 0 & 0 \\
0 & T^{2} / 2 & 0 \\
0 & 0 & T^{2} / 2 \\
T & 0 & 0 \\
0 & T & 0 \\
0 & 0 & T \\
1 & 0 & 0 \\
0 & 1 & 0 \\
0 & 0 & 1
\end{array}\right|
\end{aligned}
$$

\subsection{Gating}

In radar tracking system, association gate is defined around a predicted target position, which accumulates uncertainty of prediction, resulting from tracking filter parametrization and past performance of the tracking process. When the following relation of norm of residual error $d^{2}$ is satisfied

$$
d^{2}=\tilde{y}^{T} S^{-1} \tilde{y}(k) \leqq T_{G},
$$

measurement is considered for track update, with $\tilde{y}$ being the innovation vector described in (3) and $S$ is the innovation covariance matrix described by (4). In the literature equation (14) is known as statistical distance or Mahalanobis distance, and is a typical metric used in gating algorithms. The parameter $T_{G}$ is responsible for the effective size of the association gate and can be defined in several ways. In this paper $T_{G}$ value has been determined using chi-square statistics. This approximation can be used keeping in mind the assumed Gaussian nature of random processes used during modeling in the tracking filter. This means that the value is the sum of squares of $M$ independent Gaussian random variables with zero mean value and uniform standard deviation, where $M$ is the size of the measurement vector. ${ }^{5}$

\section{SURVEY}

Shape of the association gate is the base for association algorithm, which has a significant impact on the performance of the tracking algorithm. The article presents the influence of various factors on the shape and size of the association gates constructed using the Mahalanobis distance. 


\subsection{Shape and orientation of association gates}

First tests examines the shape of the association gate in the Cartesian system depending on the orientation of the detected object relative to the radar. For this examination assumed radar measurement error is 0.25 [deg] in azimuth $0.5[\mathrm{deg}]$ in the elevation and $15[\mathrm{~m}]$ in the distance, and the covariance of the model matrix is:

$$
Q=\left|\begin{array}{ccc}
100 & 0 & 0 \\
0 & 100 & 0 \\
0 & 0 & 100
\end{array}\right| .
$$

The results of simulation tests are shown in Figure 1 and Figure 2. Figure 1 shows the change in the angle of inclination in the X-Y plane and the size of the association gate during movement of the object from east to west. Figure 2 shows the relationship of the angle of inclination in the Y-Z plane and the size of the gate depending on the height of tracked object.
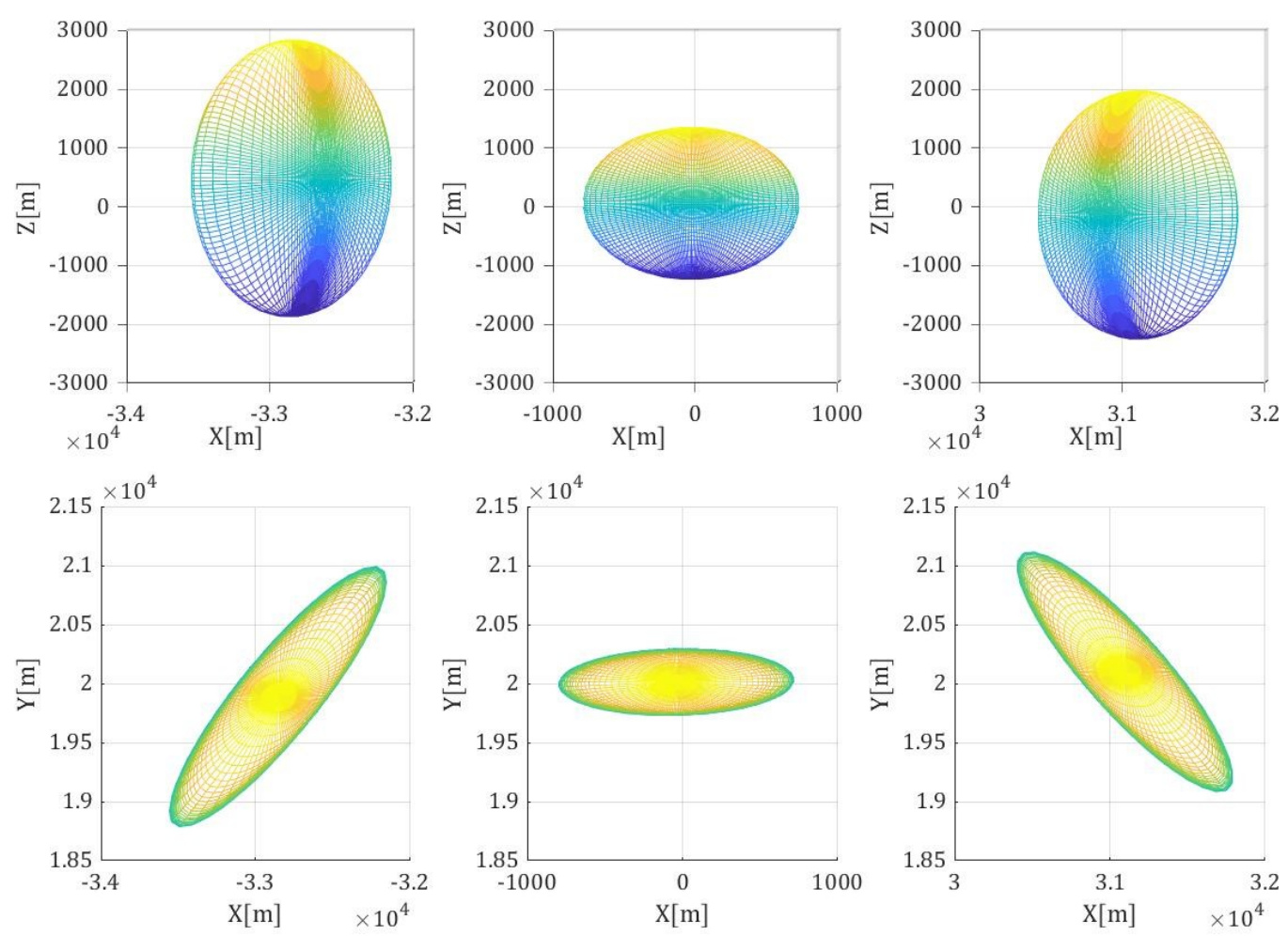

Figure 1. Shape and orientation of association gates depending on detection point in X-Y plane 

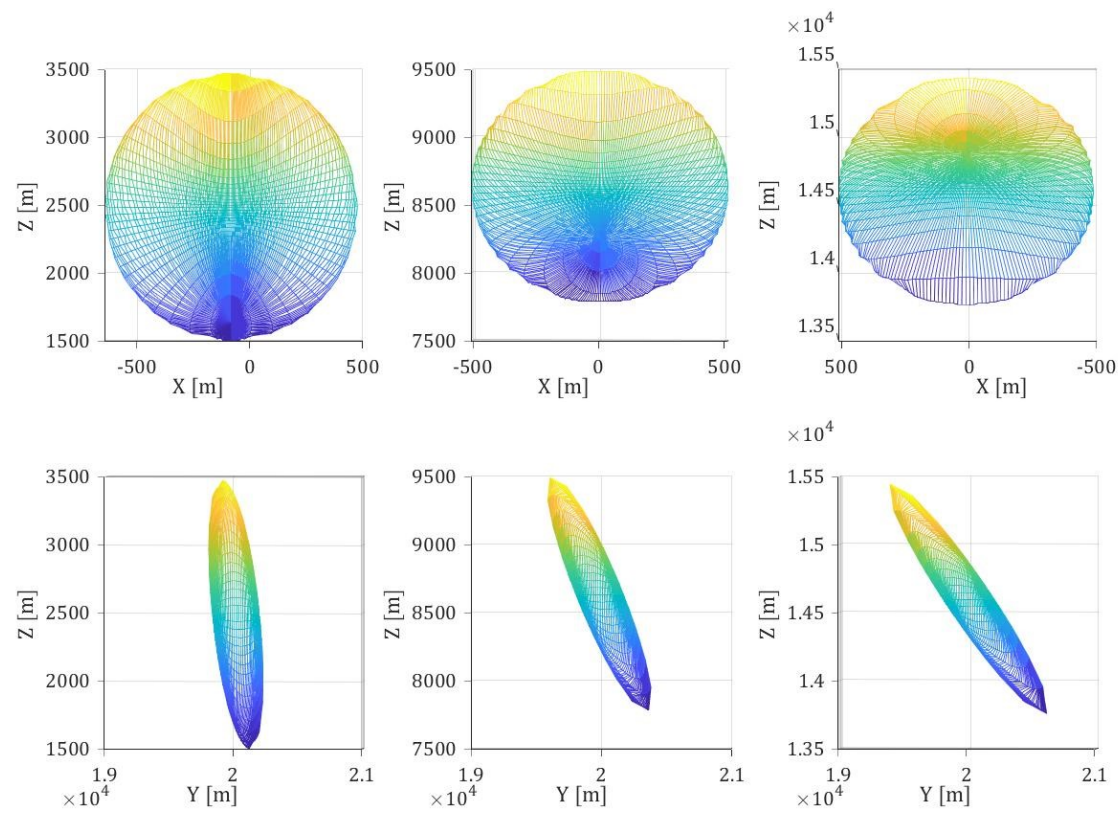

Figure 2. Shepe and orientation of associatioin gates depending on hight of detection object

Another test concerns the change in the shape of the association gates depending on the radar parameters. Assumed radar measurement error is 0.25 [deg] in azimuth 0.25 [deg] in the elevation and 15 [m] in distance.
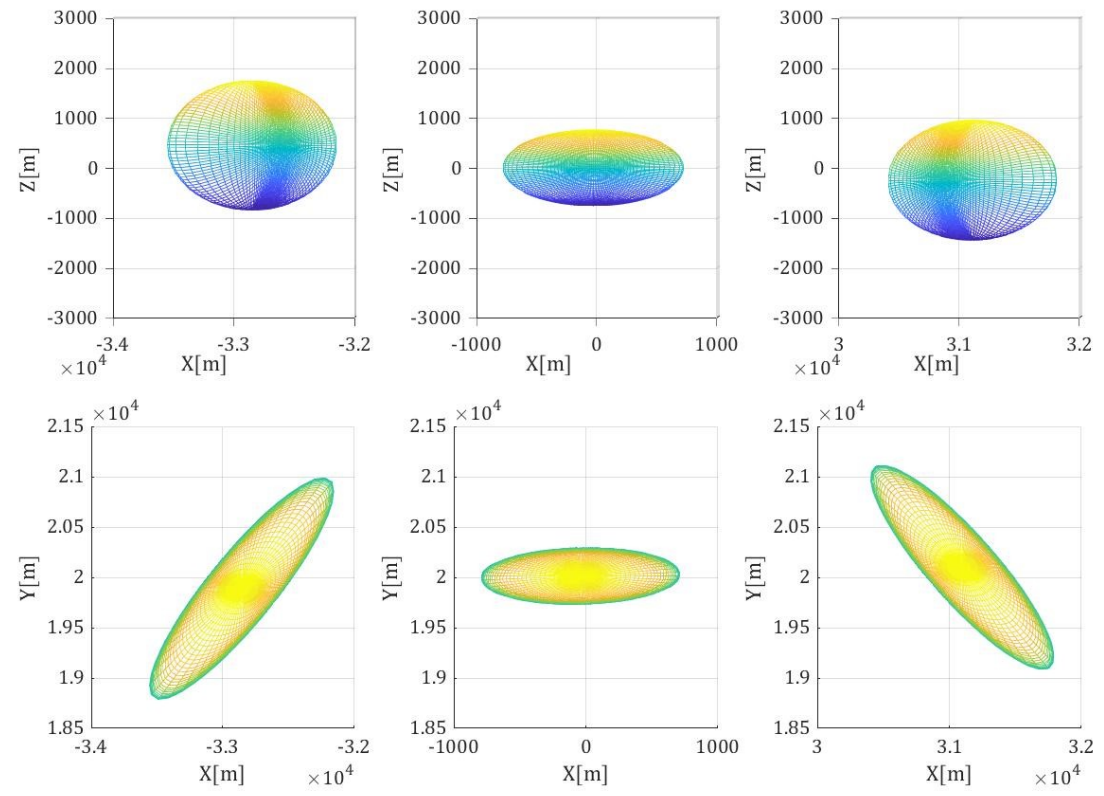

Figure 3. Shape and orientation of association gates depending on detection point in X-Y plane with various $R$ matrix

Next study concerns the impact of matrix selection on the size of the association gate. For this examination assumed covariance of the model matrix is:

$$
Q=\left|\begin{array}{lll}
5 & 0 & 0 \\
0 & 5 & 0 \\
0 & 0 & 5
\end{array}\right| .
$$



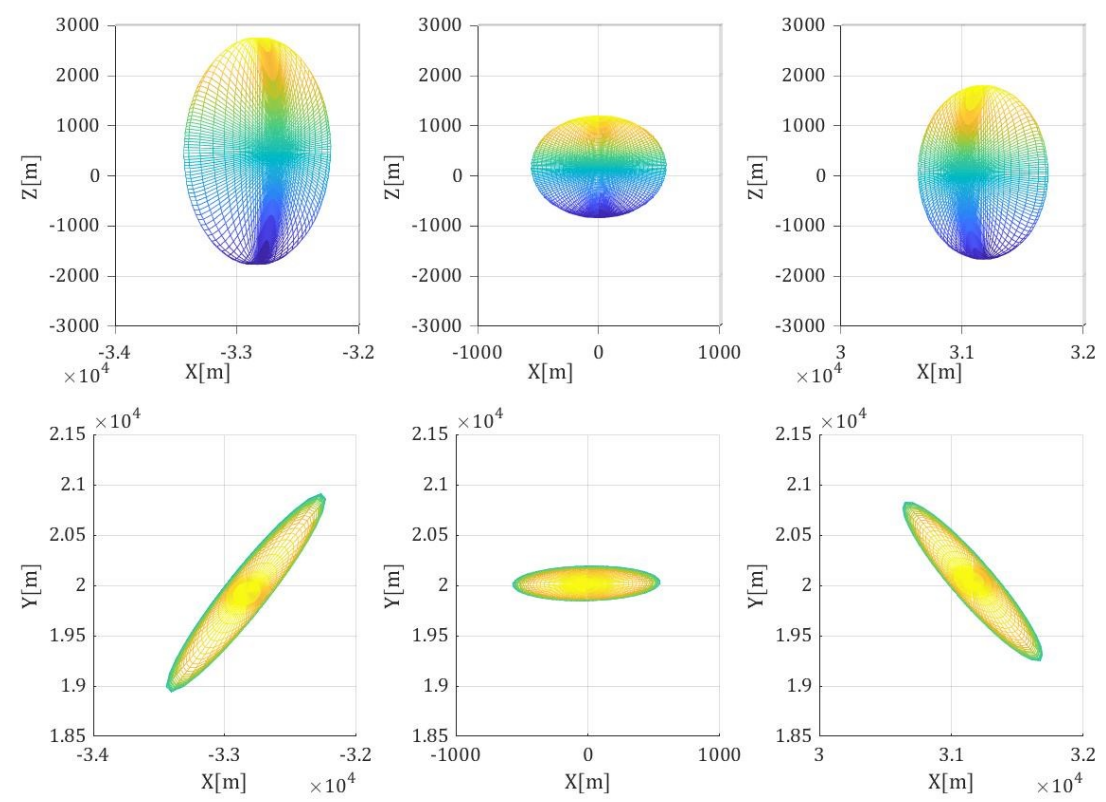

Figure 4. Shape and orientation of association gates depending on detection point in X-Y plane with various $Q$ matrix.

Results are shown in Figure 4. As we expect orientation of the association gate (direction of the axis of the large ellipse - the association gate on the X-Y plane) is closely related to a straight line perpendicular to the so-called line of sight (a straight line connecting the prediction point and radar). The angle formed by the perpendicular line with the $\mathrm{X}$ axis approximately describes the orientation of the association gate (Figure 5).

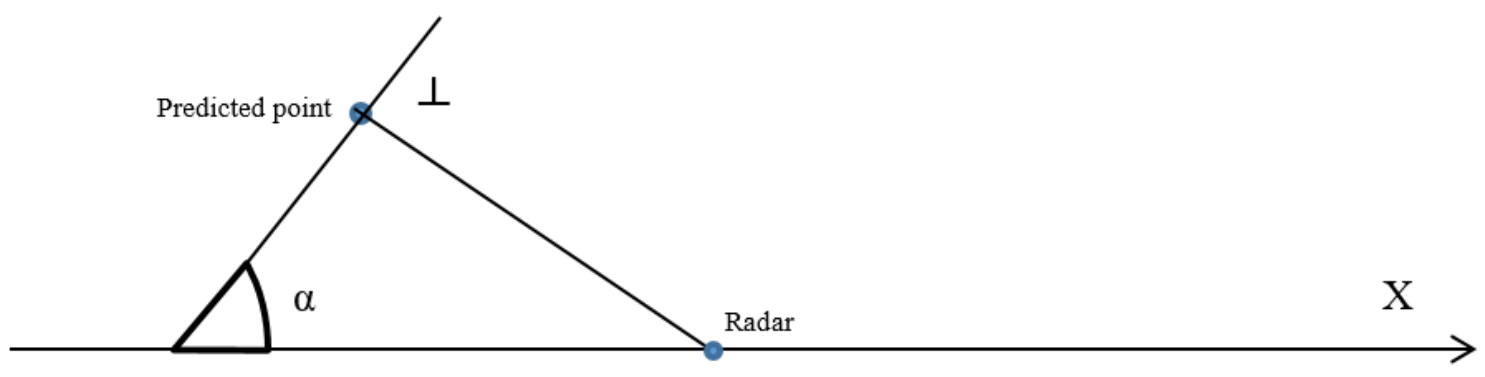

Figure 5. Geometric dependencies of the association gate orientation.

Height at which the object was detected also has a great impact on the orientation of the association gate in space. Depending on the height, the association gate is inclined to the X-Y plane, where inclination is increasing with height (Figure 2). Size of the association gate, specified in the cartesian coordinate system, is mostly influenced by the distance of the tracked object from the radar. Farther the tracked object is from the radar, the more the angle measurement errors translate into the absolute error of position measurement, which consequently enlarges the gate (Figure 1). The radar parameters themselves are another important factor affecting the shape of the gate. Comparing Figures 1 and 3, it can be seen that reducing the elevation error two times reduces the height of the association gate twice. Comparison of Figure 1. with Figure 4. allows comparision of the effect of the matrix on the shape of the association gate. Decreasing the value of matrix parameters visibly reduces the size of gate, but much less than the radar measurement error. 


\subsection{Influence of state vector on the shape of association gate}

Shape and orientation of association gates is also influenced by the object state vector. Figure 6 and 7 show impact of track direction on orientation of association gates. Figure 6 show the average value of angle of orientation for 10 simulations for opposing direction of simulated object velocity. The tests assumed that in the first case the object moves from east to west and in the second case from west to east. The object is moved only in the $\mathrm{X}$ axis with a constant position value in the $\mathrm{Y}$ axis $=6000[\mathrm{~m}]$ and a constant height of $200[\mathrm{~m}]$.

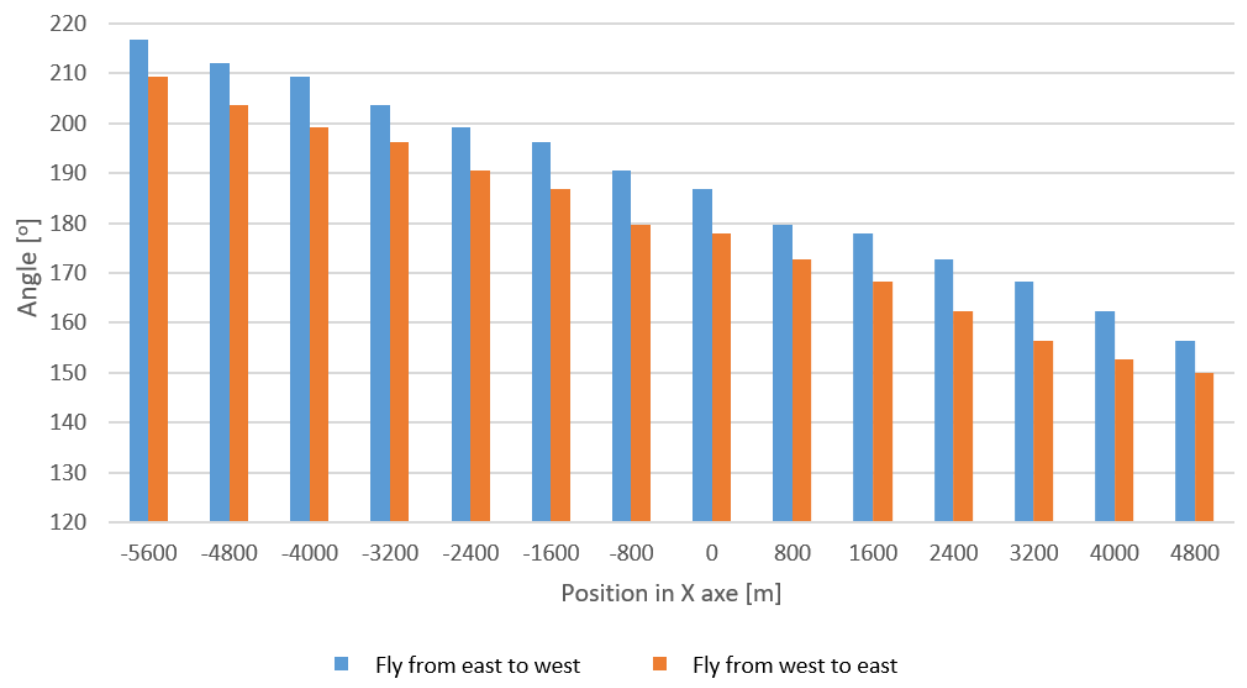

Figure 6. Shape and orientation of association gate depending on direction of tracked object move.

Figure 7 compares the average values of orientation angle of association gate from 10 simulations for object maneuver in opposite directions (course maneuver). The starting point for both maneuvers is point 1, the blue bar on the graph represents the average angle of orientation of the association gate relative to the radar for an object moving in the counterclockwise direction, and orange for the object maneuvering in a clockwise direction.
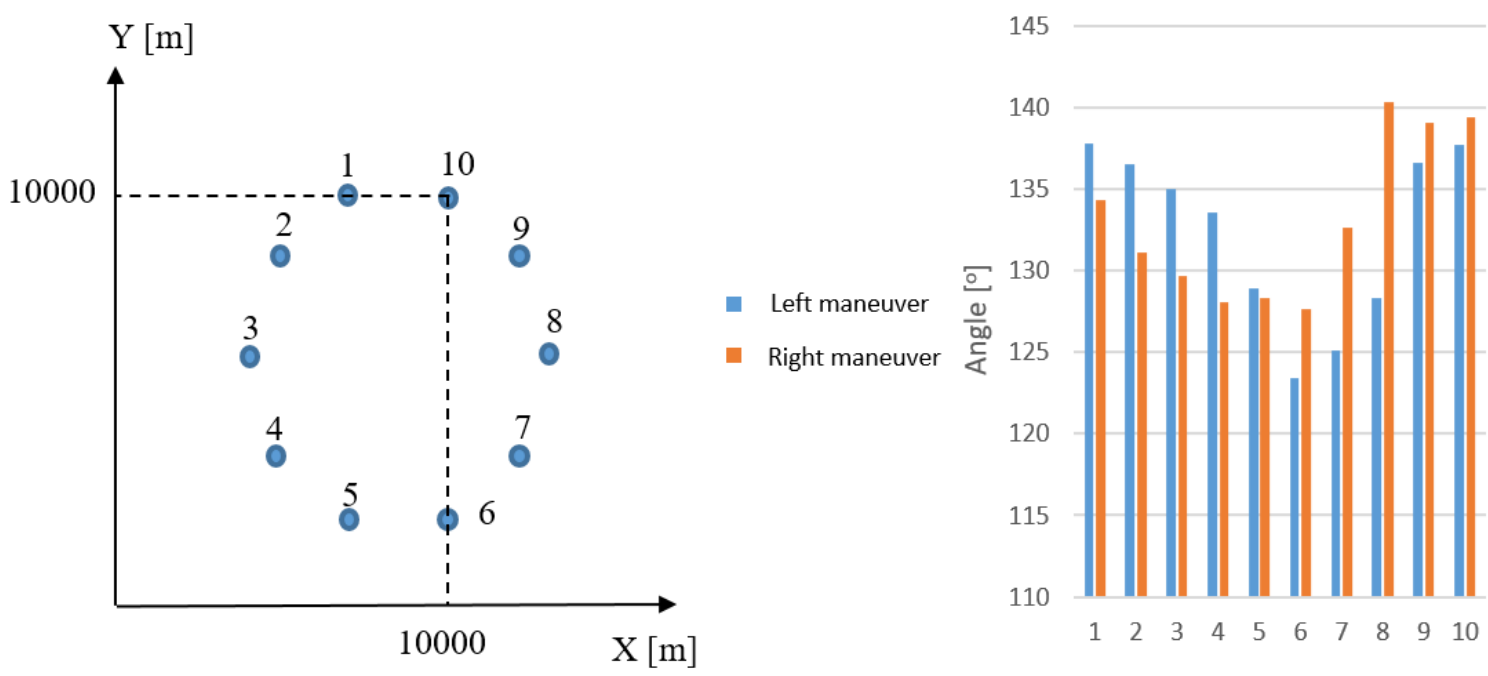

Figure 7. Shape and orientation of association gate depending on direction of tracked object maneuver.

Results presented in Figures 6 and 7 show that the orientation of the association gate is also affected by 
the state vector. Depending on the direction of movement (Figure 6) and maneuver direction (Figure 7), the orientation of the association gate slightly differs for the same object position relative to the radar.

\subsection{Impact of detection loss on association gate}

In radar tracking data loss due to lack of detection is a common problem. In this situation, the tracking algorithm extrapolates track performing track update without measurement. Such operation means also extrapolation of the state covariance matrix, which effectively results in a relative increase in value in the future innovation covariance matrix. The impact of that loss on the size of the association gate is presented in Figure 8. Figure 9 shows the reference sizes of subsequent association gates for the case without lack of detection.
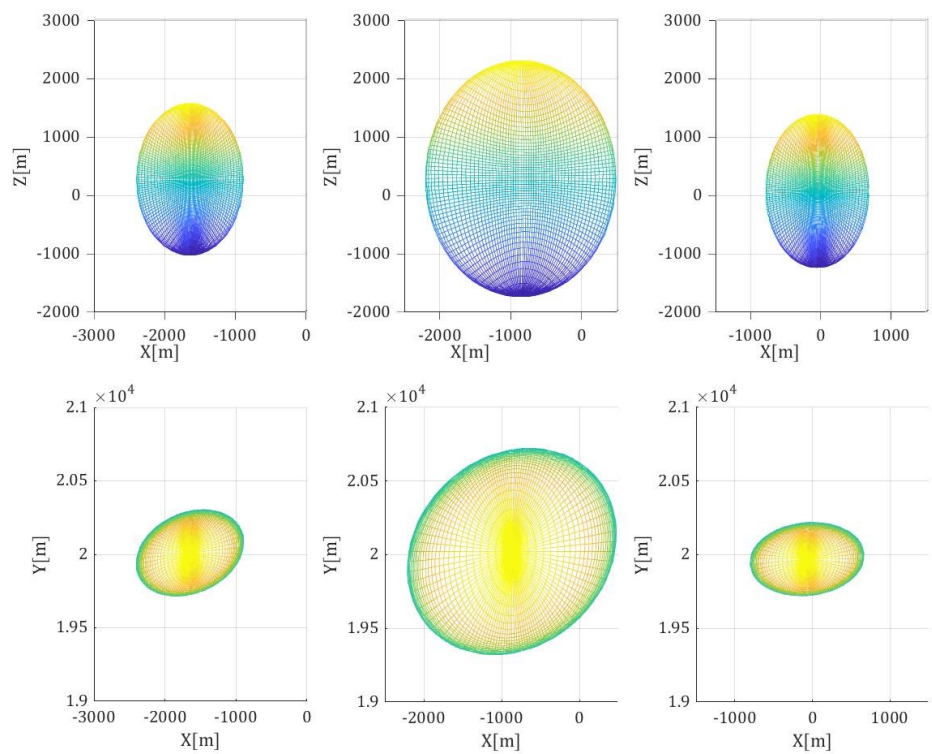

Figure 8. Shape and orientation of association gate during lack of detection of object.
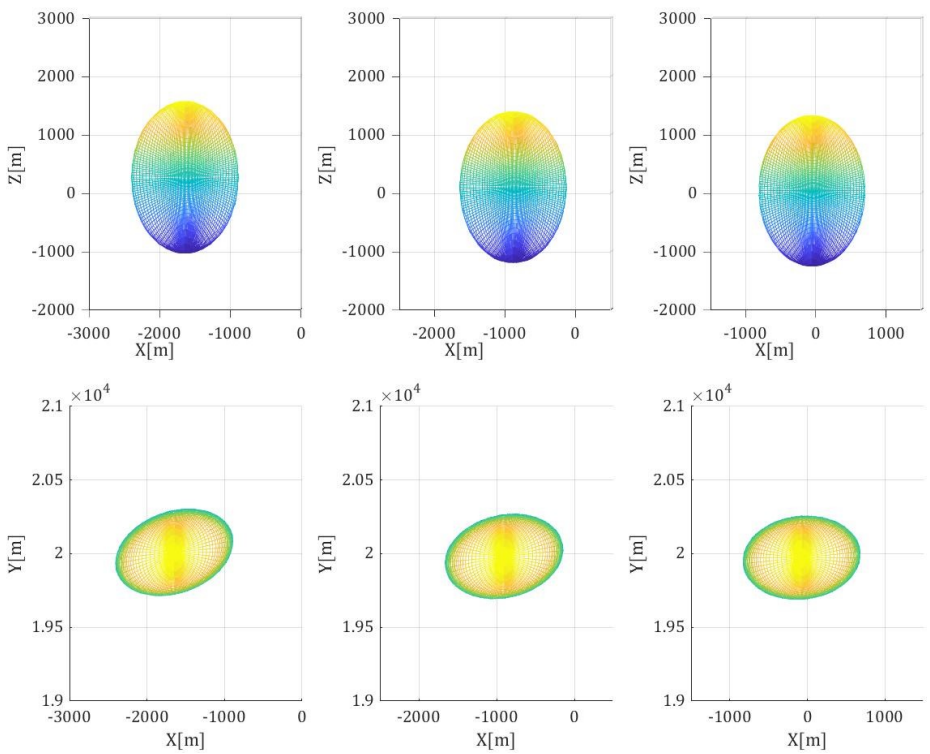

Figure 9. Shape and orientation of association gate during lack of detection of object, reference datas. 
Lack of detection almost doubled size of the gate. However, subsequent update with measurement quickly reduces association gate to regular size.

\section{SUMMARY}

In this paper the impact of selected factors on the shape and orientation of association gates based on Mahalanobis distance, is examined using computer modelling and simulation. The greatest impact on the size of the association gates have measurement errors and distance of the object to radar. Another major factor is the parametrization of the tracking filter model of dynamics of observed objects and the measurement process. Differences in the association gate orientation relative to the radar were also observed for different values of the tracked object state, where the study focused on differences in the course of object. Parametrization of the dynamics model, in the form of covariance for model noise, affects the size of the association gate, but this impact is relatively small. Article shows also differences in the gate size resulting from the track update in the case of lack of detection.

Chosen scenarios are not the only ones that can affect the shape and size of the association gate. In particular, cases of wrong parameters for tracking filter (the measurement noise in the tracking filter does not correspond to real radar measurement errors), or the case of rapid changes in the tracked object dynamics (course maneuver, speed maneuver). Subsequent articles can also analyze impact of innovation process on the size of subsequent association gates to examine how the accuracy of the prediction affects the association process.

\section{REFERENCES}

[1] Bar-Shalom, Y. and Tse, E., "Tracking in a cluttered environment with probabilistic data association," Automatica 11(5), 451-460 (1975).

[2] Reid, D., "An algorithm for tracking multiple targets," IEEE transactions on Automatic Control 24(6), 843-854 (1979).

[3] Bar-Shalom, Y., Fortmann, T. E., and Cable, P. G., "Tracking and data association," (1990).

[4] Bar-Shalom, Y. and Li, X.-R., [Multitarget-multisensor tracking: principles and techniques], vol. 19, YBs Storrs, CT (1995).

[5] Blackman, S. S., "Multiple-target tracking with radar applications," Dedham, MA, Artech House, Inc., 1986, 463 p. (1986). 